\title{
Hábitos Bucais Deletérios e Possíveis Intervenções: uma Revisão de Literatura
}

\author{
Deletery Oral Habits and Possible Interventions: a Literature Review
}

\author{
Alisson Gabriel Idelfonso Bistaffa ${ }^{\mathrm{a}}$; Paula Vanessa Pedron Oltramari ${ }^{\mathrm{a}}$; Ana Claudia Castro Ferreira Contia ; Marcio \\ Rodrigues de Almeida ${ }^{\mathrm{a}}$; Arnaldo Pinzan ${ }^{\mathrm{b}}$; Thais Maria Freire Fernandes*a
}

\author{
annopar, Programa de Pós-Graduação Stricto Sensu em Odontologia. PR, Brasil. \\ ${ }^{b}$ Universidade de São Paulo, Faculdade de Odontologia de Bauru. SP, Brasil. \\ *E-mail: thais.poleti@unopar.br
}

\begin{abstract}
Resumo
A Odontologia visa o equilíbrio e a manutenção das estruturas bucais para atingir os objetivos anatomofuncionais, musculares e estruturais. Os hábitos bucais deletérios são caracterizados como comportamentos que quebram esse equilíbrio, por meio de qualquer função anormal exercida pela musculatura orofacial. Interferem no desenvolvimento normal da oclusão e são motivos para estudos em relação as suas origens e consequências. Na Ortodontia, sabendo-se que esses comportamentos afetam a oclusão e as características ideais dos arcos dentários, a preocupação com o desenvolvimento normal da oclusão dentária motivou a realização de uma revisão de literatura sobre os hábitos bucais deletérios e o estabelecimento de uma sistematização de tratamento. Foi realizada uma revisão de literatura, nas bases de dados eletrônicas: Pubmed, SciELO e Google Acadêmico. Sendo selecionados os artigos em inglês e português, que versam sobre os fatores etiológicos envolvidos nos hábitos bucais deletérios, seu diagnóstico, suas implicações clínicas e possibilidades de tratamento. Apresentam fatores etiológicos, diagnóstico e implicações clínicas para nortear as atitudes do cirurgião-dentista diante de possíveis más oclusões advindas de um hábito. É de suma importância a identificação precoce dos aspectos clínicos e físicos presentes nos pacientes com hábitos bucais deletérios para tratamento adequado, no momento certo. Foi possível verificar que o diagnóstico correto e a intervenção precoce trazem benefícios estéticos e funcionais ao paciente, evitando-se que as más oclusões incipientes originem deformidades esqueléticas permanentes de difícil tratamento, minimizando o tratamento corretivo posterior e atribuindo maior estabilidade nos resultados alcançados.
\end{abstract}

Palavras-chave: Hábitos. Etiologia. Má Oclusão.

\begin{abstract}
Dentistry aims to balance and maintain oral structures to achieve anatomical, functional, muscular and structural objectives. Abnormal habits are characterized as behaviors that break this balance, through any abnormal function performed by the orofacial muscles. They interfere with the normal occlusion development and are grounds for studies regarding its origins and consequences. In orthodontics, it is known that these behaviors affect the occlusion and ideal characteristics of dental bows, the concern with the normal development of dental occlusion motivated a literature review on deleterious oral habits and the establishment of a treatment systemization. The aim of this study was to conduct a literature review, based on a search conducted in the electronic databases Pubmed, SciELO and Google Scholar. Articles that dealt with the etiological factors involved in abnormal oral habits, their diagnosis, clinical implications and treatment possibilities were selected. They present etiological factors, diagnosis and clinical implications to guide the dentist's attitudes towards possible malocclusions arising from a habit. It is extremely important to identify the clinical and physical aspects present in patients with harmful oral habits for appropriate treatment at the right time. Correct diagnosis and early intervention bring aesthetic and functional benefits to the patient, preventing incipient malocclusions from causing permanent skeletal deformities that are difficult to treat, minimizing subsequent corrective treatment and giving greater stability to the results achieved. A narrative literature review was carried out.
\end{abstract}

Keywords: Habits. Etiology. Malocclusion.

\section{Introdução}

A Odontologia visa o equilíbrio e a manutenção das estruturas bucais para atingir os objetivos anatomofuncionais, musculares e estruturais. Assim, na arquitetura da oclusão ideal, espera-se uma relação de interdependência e harmonia entre os dentes, as bases ósseas e a musculatura do sistema estomatognático. Os dentes ocupam sua posição de equilíbrio, em que as forças opostas, provenientes das musculaturas intrabucal (língua) e extrabucal (bochechas e lábios), neutralizam-se. A quebra desse equilíbrio muscular, por meio de qualquer função anormal (hábito deletério) exercida pela musculatura orofacial, contribuirá negativamente para $\mathrm{O}$ desenvolvimento da oclusão (ALMEIDA et al., 2000).

Define-se como hábitos bucais aquelas funções que contribuem para o estabelecimento de uma oclusão normal e favorecem a liberação do potencial de crescimento facial em toda a sua plenitude, sem desvios. Dessa maneira, essas funções exigem o uso correto da musculatura intrabucal e facial durante a respiração, deglutição, fonação, mastigação e postura. Contudo, quando as funções bucais constituem fatores etiológicos em potencial na deterioração da oclusão e na alteração do padrão normal de crescimento facial, essas são 
consideradas deletérias (SILVA FILHO; OKADA; SANTOS, 1986; MEW, 2015; BORRIE et al., 2015).

Os hábitos bucais deletérios mais relatados na literatura são: o hábito de sucção, a respiração bucal e a deglutição atípica que podem ter implicações biológicas, psíquicas e sociais. Entre as implicações biológicas se encontram as alterações dentoalveolares, esqueléticas e neuromusculares. Nas psíquicas, as alterações emocionais poderão ser tanto o fator causal do hábito como também o seu resultado, já no âmbito social, essas alterações poderão acarretar meios de exclusão da sociedade (BORRIE et al., 2015; DIMBERG et al., 2015; GUEDES et al., 2016; KNOSEL et al., 2016; OLIVEIRA et al., 2010; PRIMOZIC et al., 2013; SHARMA, BANSAL; ASOPA, 2015; ROSSATO et al., 2018; SCHMID et al., 2018). Constituem motivo para pesquisas nos meios médico, psicológico, fonoaudiológico, odontopediátrico e ortodôntico. O tema desperta atenção na tentativa de se estabelecer uma relação entre causa e efeito para determinadas más oclusões (BORRIE et al., 2015; KNOSEL et al., 2016; GERMA et al., 2016).

É de fundamental importância diagnosticar corretamente os hábitos deletérios e interceptá-los de maneira precoce para evitar, no futuro, más oclusões mais severas e, consequentemente, tratamentos ortodônticos mais complexos (BORRIE et al., 2015). Objetivou-se com o presente trabalho revisar a literatura sobre os possíveis fatores etiológicos envolvidos nos hábitos bucais deletérios, bem como o seu diagnóstico, as suas implicações clínicas e prováveis tratamentos.

\section{Desenvolvimento}

\subsection{Metodologia}

O presente estudo consiste em revisão bibliográfica. Fundamentou-se em busca realizada nas bases de dados eletrônicas: Pubmed, Scielo e Google Acadêmico. As palavraschave utilizadas foram: Habits, Etiology e Malocclusion. Foram selecionados os artigos que versavam sobre os fatores etiológicos envolvidos nos hábitos bucais deletérios, seu diagnóstico, suas implicações clínicas e possibilidades de tratamento.

\subsection{Hábito de sucção}

O sistema estomatognático possui algumas funções básicas definidas, tais como: a sucção, a mastigação, a fonoarticulação e a respiração, que desempenham um importante papel no crescimento craniofacial e na fisiologia oclusal. A função de sucção, em especial, merece considerações específicas, quando se pretende avaliar um possível rompimento deste equilíbrio, principalmente, quando se atenta para a possibilidade de tal função se prolongar com o tempo, originando um dos hábitos bucais deletérios mais comuns na infância (BORRIE et al.,2015; HENRIQUES; CRUZ, 2003; GÓIS et al., 2008; SHARMA; BANSAL; ASOPA, 2015;).

Romette (1978) enfatiza que a sucção é uma função vital, característica dos mamíferos, a qual irá suprir não somente as necessidades nutricionais (sucção nutritiva) do recémnascido como também suas necessidades emocionais (sucção não-nutritiva) e maturacionais, por meio do envio ao Sistema Nervoso Central de estímulos, tais como: o contato lábiolíngua-seio materno.

Assim sendo, os hábitos bucais de sucção constituem elementos familiares na vida cotidiana nos estágios de desenvolvimento infantil, enraizados desde a vida intrauterina, o que lhes confere um caráter singular e enternecedor, além de um certo tom de normalidade nos primeiros anos de vida. Todavia, a relação de causa e efeito entre os hábitos de sucção e a má oclusão não constitui invenção do terceiro milênio, sendo citada na literatura desde 1878 por Chandler, e tampouco restrita à Odontologia (SILVA FILHO et al., 2003; GUEDES et al.,2016).

\subsubsection{Etiologia}

As várias teorias sugerem que o hábito de sucção deve ser visto como um padrão de natureza multivariada, pois é possível que a sucção se inicie por uma razão e seja sustentada nas idades subsequentes por outros fatores, uma vez que o problema de sucção do polegar nem sempre é um sintoma único e isolado, mas, ao contrário, pode ser um grau de vários sintomas relacionados a conflitos e a instabilidade emocional, com raízes em muitas situações anteriores, como fatores culturais e determinantes socioeconômicos (GURGEL et al., 2003; BORRIE et al., 2015).

Muitos autores acreditam que a insatisfação da atividade de sucção durante os primeiros meses de vida, ou seja, durante o período de lactância, está realmente associada ao aparecimento de hábitos de sucção não nutritivos (MOFFAT,1963; FERREIRA; TOLEDO, 1967). Além disso, acredita-se que a amamentação natural possa minimizar os danos ou até mesmo evitar o hábito de sucção, pois é responsável pelo desenvolvimento motricional da cavidade bucal do bebê, prevenindo hábitos de sucção indesejáveis e alterações no padrão de atividade muscular, os quais podem interferir no desenvolvimento normal dos arcos dentários e palato duro, levando a posterior alteração oclusal (FERREIRA; TOLEDO, 1967; BORRIE et al., 2015).

\subsubsection{Diagnóstico}

O diagnóstico das más oclusões oriundas dos hábitos bucais deletérios não foge à rotina ortodôntica, exigindo um exame clínico acurado e uma anamnese completa com os pais e com a criança, de maneira cuidadosa, seguidos de exame dos modelos de gesso e de uma detalhada interpretação radiográfica. Além disso, deverão ser considerados os fatores: idade do paciente, duração, frequência, intensidade e o posicionamento do dedo ou chupeta, visto que implicará nas características clínicas e possíveis tratamentos (SEIXAS; ALMEIDA; FATTORI, 1988; BORRIE et al., 2015). 


\subsubsection{Implicações clínicas}

O tipo e a gravidade de uma má oclusão provocada pelo hábito de suç̧ão dependem de fatores relacionados, tais como: intensidade, frequência e duração (Tríade de Graber); resistência alveolar e do padrão dentofacial inerente à criança (GOÍS et al, 2008; BORRIE et al., 2015).

As alterações dentoesqueléticas mais relatadas na literatura (COZZA et al., 2005; MADIRAJU et al., 2011; ROSSATO et al., 2018; GERMA et al., 2016; KURAMAE et al., 2001, DIMBERG et al., 2015, BORRIE et al.,2015; GÓIS et al.,2008; FERES et al., 2015; SHARMA, BANSAL, ASOPA, 2015; SCHMID et al., 2018) consequentes ao hábito de sucção prolongada estão relacionadas abaixo:

a) Mordida aberta anterior: a alteração mais comum, em função do posicionamento do(s) dedo(s) impedir diretamente a erupção dos incisivos, e ao mesmo tempo, predispõe a separação dos maxilares e altera o equilíbrio nos dentes posteriores, ocorrendo maior erupção desses, o que contribui fortemente para o desenvolvimento dessa má oclusão.

b) Vestibularização dos incisivos superiores: a pressão exercida pela face interna do dedo ou pela chupeta induz os dentes a uma protrusão e/ou inclinação para vestibular.

c) Verticalização dos incisivos inferiores: associada à pressão que o dorso do dedo pode exercer; bem como a pressão exercida pela língua durante a sucção.

d) Mordida cruzada posterior: resultante da somatória de forças em desequilíbrio presentes nesse hábito, ocasionadas pelo aumento da atividade da musculatura jugal e pelo abaixamento postural da língua, com consequente atresia maxilar.

e) Interposição lingual: pode ser responsável pela perpetuação da mordida aberta anterior mesmo após o abandono espontâneo do hábito de sucção e acarretar alterações na fala, com relação à pronúncia dos sons: /t/, /d/, /n/, /l/ e dos fonemas /s/, /z/, /ch/.

\subsubsection{Tratamento}

Em síntese, pelos comentários acerca das implicações do hábito, pode-se inferir que o hábito de sucção suscita alterações morfológicas na oclusão dentária, desvios funcionais da musculatura circunjacente e envolve aspectos emocionais da criança. Todos esses fatores devem ser abordados por uma terapia multidisciplinar para que o tratamento alcance o êxito desejado, sem qualquer sequela para o paciente (SILVA FILHO,1986).

A mecanoterapia é citada desde 1930 por Lewis, e desde então, a classe odontológica vem tentando aprimorar e criar mecanismos que possam minimizar os danos causados por este hábito. Muitos autores citam que se o hábito for interrompido, na fase de dentadura decídua, há grandes chances de autocorreção da má oclusão (ALMEIDA, 1998; ESTRIPEAUT; HENRIQUES; ALMEIDA, 1989; SILVA FILHO; FREITAS; CAVASSAN, 1986). Todavia, esta correção espontânea não é dependente somente da época de interrupção do hábito, mas também da gravidade da má oclusão, da competência da musculatura perioral, do padrão dentofacial da criança e da presença de outros hábitos deletérios (SILVA FILHO; FREITAS, CAVASSAN, 1986).

Os dispositivos empregados na tentativa de se obter a correção são vários, desde os mais imponentes e menos utilizados, como os dispositivos mecânicos sobre os dedos, tais como: luvas de box, algemas nos braços e outros tipos de contenção, além dos dispositivos químicos, como: babosa amarga, pimenta, etc. (MOFFAT, 1963; JOHNSON, 1993). Porém, na grande maioria dos casos, diversos autores recorrem aos clássicos aparelhos mecânicos para interromper o hábito de sucção e possibilitar a consequente correção da mordida aberta anterior. Esses aparelhos intrabucais, simples impedidores passivos, constituídos por grades palatinas, funcionam como recordatórios para a criança. A grade palatina possui uma finalidade dupla: tornar impraticável a sucção do dedo e dificultar a interposição da língua. Retirada a força mecânica do dedo e da língua contra os dentes, a ação de moldagem da musculatura labial incumbe-se de corrigir a alteração dento-alveolar (POETSCH, 1968; SILVA FILHO, 1986; BONI, 1997; ALMEIDA, 1998; GURGEL, 2003). Uma vez corrigido o desvio morfológico, a grade palatina deverá ser substituída por uma placa de Hawley para contenção (SILVA FILHO, 1986). Além disso, é ressaltado que na presença da mordida cruzada posterior, o tratamento deve ser iniciado com a mecânica para descruzar a mordida (SILVA FILHO, 1986; GURGEL, 2003).

\subsection{Hábito de respiração bucal}

Na normalidade fisiológica, a respiração deve ser realizada predominantemente via nasal, para que o ar inspirado chegue aos pulmões umedecido, aquecido e filtrado. Apenas em momentos de maior demanda de oxigênio, como durante grandes esforços físicos, estabelece-se uma respiração mista, nasal e bucal. Porém, diante de obstruções presentes, ao longo das vias aéreas, a criança forçosamente passa a respirar pela via alternativa, a cavidade bucal (ALMEIDA, 2000). A respiração bucal é um hábito deletério que representa uma disfunção respiratória (FERREIRA, 1997). Pacientes com obstrução nasal crônica se tornam respiradores bucais, o que leva a deformidades faciais, principalmente, durante a fase de crescimento (MOCELLIN; CIUFFI, 1997).

\subsubsection{Etiologia}

Os hábitos bucais, considerados deletérios, ocasionam a perda do equilíbrio do padrão do funcionamento muscular, que sendo induzido por arcos reflexos, torna sua ação duradoura. A função respiratória está diretamente relacionada ao desenvolvimento craniofacial. Assim sendo, a respiração bucal também é considerada um hábito bucal deletério, quando presente em casos como: o de esforços físicos demasiados; 
por obstruções das vias aéreas superiores e, também, por causas não obstrutivas (DI FRANCESCO, 1999).

A respiração por esforço físico não está incluída nas causas patológicas. Porém, as causas de obstrução das vias aéreas superiores são patológicas e englobam: hipertrofia de cornetos; trauma nasal; hipertrofia adenoamigdaliana; malformações nasais; insuficiência alar, atresia de coanas, pólipos, tumores da cavidade nasal e rinofaringe; hipertrofia de tonsilas palatinas e adenoides; rinites e desvio de septo, sendo as três últimas citadas, mais frequentemente na prática odontológica (ALMEIDA; URSI, 1990; NOVAES, 1993; MOCELLIN; CIUFFI, 1997).

\subsubsection{Diagnóstico}

A etiologia da respiração bucal é complexa e variada (ALMEIDA; URSI, 1990; NOVAES, 1993; MOCELLIN; CIUFFI, 1997; DI FRANCESCO, 1999), em virtude disso, diferentes métodos de avaliação são relatados.A avaliação clínica pode ser feita a partir de alguns exames, que são realizados com o paciente sentado corretamente, com a cabeça bem posicionada sobre a coluna vertebral para que não ocorra qualquer desvio de postura que altere as observações. A análise deve ser iniciada antes mesmo que o paciente perceba. Os respiradores bucais, mesmo que consigam respirar pelo nariz, não alteram a forma das narinas externas por não terem controle dos músculos alares, e, muitas vezes, contraem os orifícios nasais na inspiração por dificuldade de obter ar suficiente por essa via, além de manter os lábios entreabertos durante a respiração relaxada. Durante esse período, os olhos, o tórax e o abdome são observados, bem como as alterações de expressão, comportamento ou movimento físico exagerado (NOVAES; VIGORITO, 1993).

Os métodos de observação contribuem para o diagnóstico e podem ser complementados com a análise por meio de espelhos de dupla face durante a expiração. Posiciona-se sobre o lábio superior e se avalia a área embaçada. Se a criança for um respirador bucal, a superfície inferior irá embaçar, porém se esta for respirador nasal, será a superfície superior que embaçará (NOVAES; VIGORITO, 1993).

São várias as etiologias da respiração bucal, assim sendo, o diagnóstico final só pode ser fornecido por um especialista, o otorrinolaringologista (ALMEIDA; URSI, 1990; NOVAES, 1993; MOCELLIN; CIUFFI, 1997; DI FRANCESCO, 1999).

\subsubsection{Implicações clínicas}

a)“Fácies adenoidea": o indivíduo apresenta rosto alongado e estreito, olhos caídos, olheiras profundas, sulcos genianos marcados, ou seja, expressão inerte em virtude da incapacidade do fluxo aéreo nasal (ALMEIDA; URSI, 1990; NOVAES, 1993; MOCELLIN; CIUFFI, 1997).

b) Narinas pequenas ou pouco desenvolvidas: pela falta de passagem de ar pela cavidade nasal, portanto, perda progressiva da função com consequente degeneração (ALMEIDA; URSI, 1990; NOVAES, 1993; MOCELLIN; CIUFFI, 1997). c) Lábios entreabertos e tonicidade labial alterada: em função da necessidade de assegurar a abertura de uma via aérea bucal, a criança permanece com os lábios entreabertos não exercendo função adequada, tornando-se na maioria das vezes hipotônicos e ressecados (ALMEIDA; URSI, 1990; NOVAES, 1993; MOCELLIN; CIUFFI, 1997).

d) Palato ogival: a posição de repouso da língua para trás e sobre o assoalho da boca, para permitir a passagem do ar, não se contrapõe às forças laterais exercidas sobre a maxila pelos músculos bucinadores, alterando dests forma a altura da abóbada palatina. Além disso, a deficiência da passagem de ar pela cavidade nasal também não propicia o crescimento maxilar no sentido transversal (ALMEIDA; URSI, 1990; NOVAES, 1993; MOCELLIN; CIUFFI, 1997).

e) Mordida cruzada posterior: durante a inspiração e expiração do portador do hábito o ar só passa pela cavidade bucal, tendo como consequência um aumento da pressão aérea intrabucal. O palato, portanto, vai se modelando e aprofundando e a característica desta atresia maxilar é a mordida cruzada posterior (ALMEIDA; URSI, 1990; NOVAES, 1993; MOCELLIN; CIUFFI, 1997).

f) Vestibuloversão dos incisivos superiores: pela falta do selamento labial, o lábio superior não exerce sua função no equilíbrio labiolingual para o posicionamento dentário. A conformação atrésica da maxila também induz a protrusão dos incisivos superiores (ALMEIDA; URSI, 1990; NOVAES, 1993; MOCELLIN; CIUFFI, 1997).

g) Verticalização dos incisivos inferiores: a mudança na postura para assegurar a abertura de uma via aérea bucal faz com que os lábios fiquem entreabertos, a mandíbula seja deslocada para baixo e para trás, e a língua repousando mais inferior e anteriormente. Assim sendo, permite que uma ação muscular mais inferior e de maior tonicidade exerça pressão nas faces vestibulares dos incisivos inferiores, verticalizandoos (ALMEIDA; URSI, 1990; NOVAES, 1993; MOCELLIN; CIUFFI, 1997).

\subsubsection{Tratamento}

O tratamento da respiração bucal será melhor sucedido quanto mais cedo se intervir, em função de seus efeitos deletérios durante o processo do crescimento facial e, por isso, torna-se de vital importância o seu diagnóstico e o envolvimento de profissionais de áreas afins, tais como o otorrinolaringologista, o cirurgião-dentista e o fonoaudiólogo (GURGEL et al., 2003).

Os pacientes com respiração bucal, frequentemente, possuem deglutição atípica, um hábito bucal deletério que se processa quando há uma quebra do equilíbrio entre os músculos periorais, mastigadores e a língua (KURAMAE et al., 2001; BICALHO, MOTTA, VICENTE L, 2006).

\subsection{Hábito de deglutição atípica}

A deglutição normal é um processo muito complexo e que envolve ação muscular antagônica e sinérgica (FERREIRA, 
1967). Assim, a deglutição envolve músculos linguais da expressão facial e da mastigação. Quando esta sinergia é quebrada, surge a deglutição atípica que é um hábito bastante comum, caracterizada pela projeção da língua entre os dentes durante o ato de engolir e no repouso (MATOS et al., 2001).

A deglutição atípica exige um grande esforço dos músculos da expressão facial, promove posições atípicas da língua que desfavorece todo o quadro da deglutição, rompendo com toda a harmonia muscular da cavidade bucal e repercutindo negativamente em seus componentes (KURAMAE et al., 2001).

\subsubsection{Etiologia}

As causas mais prováveis da deglutição atípica são: desequilíbrio do controle nervoso; tonsilas inflamadas; língua posicionada anteriormente; (RODRIGUES, 1998; ALMEIDA, 1998); macroglossia; anquiloglossia; freio lingual anormal; perdas precoces dentais e diastemas anteriores; presença de mordida aberta; fatores simbióticos (sucção de dedo e respiração bucal) e hábitos bucais inadequados na primeira infância (amamentação artificial e tempo de amamentação inadequado) (SEIXAS, 1998; MATOS et al. 2001).

\subsubsection{Diagnóstico}

Para diagnosticar uma deglutição atípica se tem que observar alguns aspectos no paciente durante o ato da deglutição (independente da técnica estabelecida para o diagnóstico), tais como: posicionamento atípico da língua; ausência de contração dos masséteres; participação da musculatura perioral com pressionamento do lábio e movimentos com a cabeça; sopro ao invés de sucção; tamanho e tonicidade da língua; cuspir ou acumular saliva ao falar; baba noturna; dificuldades na ingestão de alimentos sólidos e desvios na fonação. Consequentemente, identifica-se se o paciente apresenta deglutição atípica de língua e/ou de lábio (FLETCHER; CASTEEL; BRADLEY,1991).

\subsubsection{Implicações clínicas}

A deglutição atípica se origina basicamente de um desequilíbrio da musculatura entre a cinta muscular perioral e a língua. Podendo ser devido ao posicionamento incorreto do lábio e/ou da língua (KURAMAE et al., 2001).

Com pressão atípica de lábio (interposição labial):

a) Vestibularização dos incisivos superiores e lingualização dos inferiores: o selamento da parte anterior da cavidade bucal não é feito pelo simples contato do lábio superior com o inferior, mas pela forte contração do lábio inferior interpondose entre os incisivos superiores e inferiores. Os inferiores, portanto, vão se inclinando para lingual e apinhando, enquanto os incisivos superiores vão se vestibularizando (KURAMAE et al., 2001).

b) Lábio superior hipotônico: por não participar da deglutição se torna cada vez mais hipotônico, dando aspecto de lábio curto (KURAMAE et al., 2001).

c) Hipertonicidade do lábio inferior: pela sua grande participação se torna cada vez mais hipertônico, assim como também os músculos do mento ( KURAMAE et al., 2001).

d) Sobressaliência e sobremordida: a perda de contato funcional anterior favorece a extrusão dental, aumentando a sobressaliência e a sobremordida (KURAMAE et al., 2001).

Com pressão atípica da língua:

A língua, durante a deglutição exerce pressão nos dentes anteriores por lingual ou entre esses. Apresenta as deformidades:

a) Mordida aberta anterior: a pressão é realizada na região anterior e a deglutição é efetuada com os dentes desocluídos, ficando a língua interposta entre os dentes. Apresenta como consequência a condição de mordida aberta anterior (KURAMAE et al., 2001; NOBRE et al, 2004; BORRIE et al., 2015).

b) Vestibuloversão dos incisivos superiores e/ou inferiores: quando a língua, além de se interpor entre os dentes anteriores, também exerce pressão anterior, essa faz com que os incisivos superiores e/ou inferiores exibam uma severa inclinação vestibular (KURAMAE et al., 2001; NOBRE et al, 2004; BORRIE et al., 2015).

c) Mordida cruzada anterior ou posterior: em função da quebra do equilíbrio muscular entre a língua e músculos da bochecha (; KURAMAE et al., 2001; NOBRE et al, 2004; BORRIE et al., 2015).

d) Alteração na fonação: causado pelo desequilíbrio no sistema "lábios-língua-dentes" (FLETCHER CASTEEL, BRADLEY, 1961; KURAMAE et al., 2001; BORRIE et al., 2015).

\subsection{Tratamento}

Assim, é de vital importância o diagnóstico clínico por meio da anamnese e do exame clínico, e o tratamento deve ser composto pelo aparelho respectivo e por exercícios que envolvam a musculatura peribucal. Além disso, a deglutição atípica não se constitui patologia que possa ser trabalhada adequadamente com uma dada técnica, por um único profissional, mas sim por uma equipe multidisciplinar respeitando as peculiaridades de cada caso (GURGEL et al., 2003).

As más oclusões provenientes do hábito bucal deletério devem ser tratadas dentro de um contexto multidisciplinar, em que estão envolvidos ortodontistas, odontopediatras, fonoaudiólogos, médicos, psicólogos e outros profissionais, os quais devem ser requeridos de acordo com as necessidades de cada caso. $\mathrm{O}$ sucesso do tratamento requer a avaliação minuciosa dos aspectos referentes à duração, intensidade e frequência do hábito, aliadas ao padrão facial do paciente, idade e a vontade e colaboração do mesmo e dos familiares, estabelecendo uma terapia que sempre resguarde os aspectos emocionais (GURGEL et al., 2003). 
Figura 1 - Fluxograma ilustrativo, compilando as informações referentes à etiologia, diagnóstico, implicações clínicas e tratamento de cada hábito

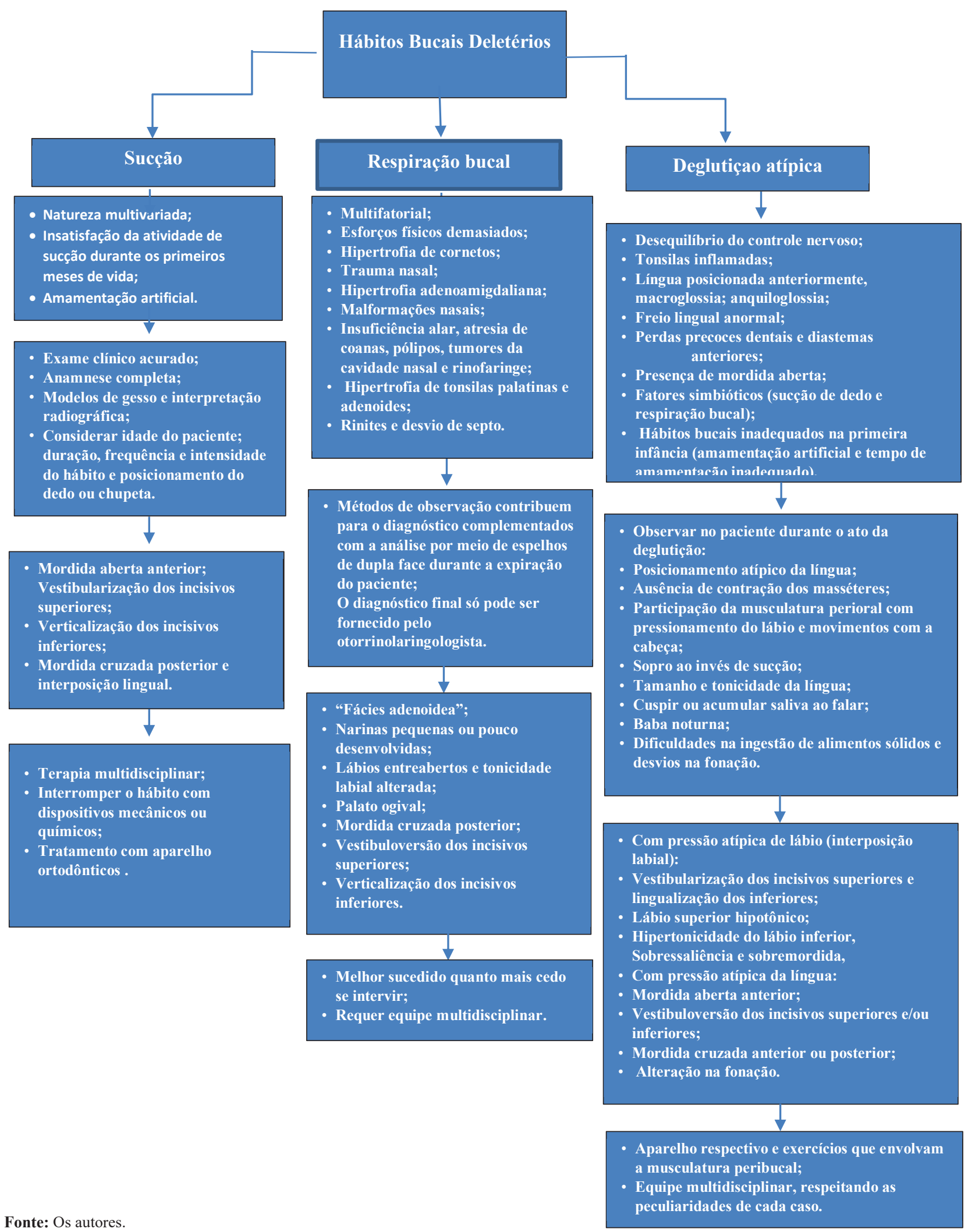

Fonte: Os autores.

e diagnosticar os aspectos clínicos e físicos presentes nos

\section{Conclusão}

Foi possível concluir que é de suma importância que o cirurgião-dentista, ainda em uma fase precoce, saiba identificar pacientes portadores de hábitos bucais deletérios, para que seus pacientes recebam o tratamento adequado no momento 
certo e consigam, assim, minimizar os distúrbios ao sistema estomatognático. Um diagnóstico correto e uma intervenção precoce podem trazer grandes benefícios estéticos e funcionais ao paciente, evitando-se que as más oclusões incipientes, de origem dentoalveolar, venham a dar origem a deformidades esqueléticas permanentes de difícil tratamento, minimizando o tratamento corretivo posterior e atribuindo uma maior estabilidade nos resultados alcançados.

\section{Referências}

ALMEIDA R.R. et al. Etiologia das más oclusões - causas hereditárias e congênitas, adquiridas gerais, locais e proximais (hábitos bucais). Rev. Dent. Press Ortodon. Ortop. Facial. v.5, n.6, p.107-129, 2000.

ALMEIDA R.R. et al. Mordida aberta anterior -considerações e apresentação de um caso clínico. Rev. Dent. Press Ortodon. Ortop. Facial, v.3, n.2, p.17-29,1998.

ALMEIDA R.R.; URSI W.J.S. Anterior open bite: etiology and treatment. Orthodontics oral heath, Canada's Leading Dent. J., v.80, n.1, p.27-31, 1990.

BICALHO P.G; MOTTA A.R; VICENTE L.C.C. Avaliação da deglutição em crianças respiradoras orais. Rev. CEFAF, v.8, n.1, p.50-55, 2006.

BONI R.C; VEIGA M.C.F.A; ALMEIDA R.C. Comportamento da mordida aberta anterior, após a remoção do hábito de sucção. J. Bras. Ortod. Ortop. Maxilar, v.2, n.12, p.35-40, 1997.

BORRIE F.R.P. et al. Interventions for the cessation of nonnutrive sucking habits in children (Review). Cochrane Database Syst. Rev., n.3, 2015. doi: 10.1002/14651858.CD008694.pub2.

CHANDLER T.J. Thumb-sucking in childhood as a cause of subsequent irregularity of the teeth. Dental Cosmos, v.99, n.7, p.565-570,1878.

COZZA P. et al. Sucking habits and facial hyperdivergency as risk factors for anterior open bite in the mixed dentition. Am. J. Orthod. Dentofacial Orthop., v.128, p.517-519, 2005.

DI FRANCESCO, R.C. Respirador bucal: a visão do otorrinolaringologista. J. Bras. Ortodon. Ortop. Facial, v.1, n.1, p.56-60, 1999.

DIMBERG L. et al. Prevalence and change of malocclusions from primary to early permanent dentition: a longitudinal study. Angle Orthod., v.85, n.5, p.728-34, 2015. doi: 10.2319/080414542.1 .

ESTRIPEAUT, L.H.; HENRIQUES, J.F.C.; ALMEIDA, R.R. Hábito de sucção do polegar e má oclusão- apresentação de um caso clínico. Rev. Odontol. USP, v.3, n.2, p.371-376, 1989.

FERES M.F.N. et al. Effectiveness of the open bite treatment in growing children and adolescents. A systematic review. Euro. J. Orthod., v.38, n.3, p.1-14, 2015.

FERREIRA, M.I.D.T.; TOLEDO, O.A. Relação entre tempo de aleitamento materno e hábitos bucais. Rev ABO Nac. v.5, n.6, p.317-320, 1967.

FLETCHER S.G; CASTEEL R.L; BRADLEY D.P. Tongue thrust-swallow speech: articulation and age. J. Speech Hear Disord., v.26, n.3, p.201-208, 1961. doi: 10.1044/jsd.2603.201

GERMA A., et al. Early risk factors for posterior crossbite and anterior open bite in the primary dentition. Angle Orthod., v.86, p.832-838, 2016.

GÓIS E.G.O. et al. Influence of nonnutritive sucking habits, breathing pattern and adenoid size on the development of malocclusion. Angle orthod., v.78, n.4,p.647-54, 2008.

GUEDES, R.S. et al. Assessing individual and neighborhood social factors in child oral health-related quality of life: a multilevel analysis. Quality Life Res., v.23, p.2521-2530,2014.

GURGEL, J.A. et al. A terapia multidisciplinar no tratamento da respiração bucal e do hábito prolongado de sucção digital ou de chupeta. Rev Dent Press Ortodon Ortop Facial, v.8, n.3, p.8191,2003.

HENRIQUES, J.F.C. et al. Sucção digital: implicações ortodônticas e estabilidade a longo prazo de um caso tratado precocemente. Rev. Clin. Ortodon. Dental Press, v.2, n.2, p.3750, 2003.

JOHNSON, E.D.; LARSON, B.E. Thumb-sucking: literature review. J. Dent. Child., v.60, n.4, p.385-391, 1993.

KNOSEL, M. et al. Interaction between deglution, tongue posture, and malocclusion: a comparison of intraoral compartment formation in subjects with neutral occlusion or different types of malocclusion. Angle Orthod., v.86, n.5, p.697-705, 2016. doi: 10.2319/101615-699.1.

KURAMAE, M. et al. Uma proposta de classificação para deglutição atípica com interposição lingual. J. Bras. Ortodon. Ortop. Facial, v.6, n.33, p.205-212, 2001.

LEWIS, S. J. Thumb-sucking a cause of malocclusion in the deciduos teeth. J. Am. Dent. Assoc., v.17, p.1060-1073, 1930.

MADIRAJU, G.S.; HARIKA L. Effectiveness of appliance therapy in reducing overjet and open bite associated with thumb sucking habit. Minerva Stomatol., v.60, p.333-338, 2011

MATOS, M.J.F. et al. Avaliação da deglutição atípica em crianças de 4 a 7 anos em rede escolar pública. J. Bras. Ortodon. Ortop. Facial, v.6, n.34, p.293-301,2001.

MEW, J. The influence of the tongue on dentofacial growth. Angle Orthod, v.85, n.4, p.715, 2015. doi: 10.2319/angl-85-04715-715.1

MOCELLIN L.; CIUFFI T. Alterações oclusais em respiradores bucais. J. Bras. Ortodon. Ortop. Maxilar, v.2, n.7, p.45-48, 1977.

MOFFAT, J.B. Habits and their relation to malocclusion. Australian Dent. J., v.8., p.44-49, 1963.

MONTALDO, L. et al. Effects of feeding on non-nutritive sucking habits and implications on occlusion in mixed dentition. Int. J. Paediatr. Dent., v.21, p.68-73, 2011.

NOBRE D.G. et al. A integracäo entre a fonoaudiologia e a odontologia no respirador bucal: a relação com maloclusäo do tipo Classe II de Angle e o seu tratamento. Rev. Paul. Odontol., v.2, n.26, p.4-11, 2004.

NOVAES M.S.P.; VIGORITO J.W. Respiração bucal: aspectos gerais e principais metodologias empregadas para avaliação. Ortodontia, v.26, n.3, p.43-52,1993.

OLIVEIRA A.C. et al. Feeding and nonnutritive sucking habits and prevalence of open bite and crossbite in children/adolescents with down syndrome. Angle Orthod., v.80, n.4, p.748-53, 2010. doi: 10.2319/072709-421.1.

POETSCH, H. Hábitos orais. ABO/GB Rev. Bras. Odontol., v.25, n.150, p.21-25,1968.

PRIMOZIC, J. et al. Influence of sucking habits and breathing pattern on palatal constriction in unilateral posterior crossbite: a controlled study. Eur J. Orthod., v.35, n.5, p.706-12, 2013. doi: 10.1093/ejo/cjs086

RODRIGUES, J. Deglutição atípica. J. Bras. Ortodon. Ortop. 
Maxilar, v.3, n.14, p.27-31,1988.

ROMETTE, D. Facteurs neuro-musculaires et morphogenese dês árcades dentaires. Rev. Orthop. Dent. Facial, n.12, p.265280,1978 .

ROSSATO, P.H. et al. Dentoalveolar effects produced by different appliances on early treatment of anterior open bite: a randomized clinical trial. Angle Orthod., v.88, n.6, p.684-91, 2018. doi: 10.2319/101317-691.1

SCHMID, K.M. et al. The effect of pacifier sucking on orofacial structures: a systematic literature review. Prog. Orthod., v.19, n.1, p.8, 2018. doi: 10.1186/s40510-018-0206-4.

SEIXAS, C.A.O; ALMEIDA, E.F; FATTORI, L. Diagnóstico, prevenção e tratamento precoce para hábitos bucais deletérios. $J$.
Bras. Ortod. Ortop. Maxilar., v.3, n.14, p.53-60,1988.

SHANTANU S.; BANSAL A.; ASOPA K. Prevalence of oral habits among eleven to thirteen years old children in Jaipur. Int. J. Clin. Pediatr. Dent., v. 8, n.3, p.208-210, 2015.

SILVA FILHO, O.G. et al. Hábitos de sucção e má oclusão: epidemiologia na dentadura decídua. Rev. Clin. Ortodon. Dental Press, v.2, n.5, p.57-74, 2003.

SILVA FILHO, O.G.; OKADA, T.; SANTOS, S.D. Sucção digital - abordagem multidisciplinar: ortodontia $\mathrm{x}$ psicologia $\mathrm{x}$ fonoaudiologia. Estomat Cult, v.16, n.4, p.61-71,1986.

SILVA FILHO, O.G; FREITAS, S.F; CAVASSAN, A.O. Hábitos de sucção: elementos passíveis de intervenção. Estomat Cult, v.16, n.4, p.61-71, 1986. 